\title{
MODELAÇÃO GEOLÓGICA DO ENCHIMENTO SEDIMENTAR PLISTO- HOLOCÉNICO DA PALEOLAGUNA DA PEDERNEIRA
}

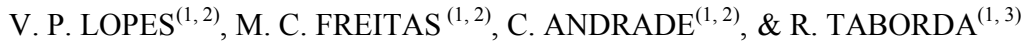

Resumo:

\begin{abstract}
A reconstrução paleoambiental das zonas húmidas litorais portuguesas constitui um elemento fundamental para o conhecimento da história evolutiva da zona costeira num passado geológico recente (Quaternário). Com a intenção de contribuir para o melhoramento do modelo de evolução da várzea da Nazaré (paleolaguna da Pederneira) durante o Plistocénico final e o Holocénico, procedeu-se à determinação da geometria e volumetria das unidades constituintes do seu enchimento sedimentar, aplicando modelação geológica tridimensional. Para esse efeito, utilizou-se a informação disponível, a qual permitiu definir três unidades litoestratigráficas (fluvial basal, marinho/lagunar e fluvial recente) e aplicou-se um modelo geomorfológico concetual ao conhecimento geomorfológico/geológico da área de estudo, o qual se mostrou imprescindível para ultrapassar a insuficiência de dados de base e a sua distribuição não uniforme na área de estudo.

Recorrendo a ferramentas de interpolação espacial, foi definida a paleosuperfície de acomodação do enchimento sedimentar plisto-holocénico, bem como as paleosuperficies que limitam as unidades constituintes daquele enchimento. Através de modelação geológica tridimensional, foi possível determinar a geometria e a volumetria total da paleolaguna da Pederneira bem como das unidades litoestratigráficas, e aferir a verosimilidade dos valores produzidos através do cálculo da erosão média da superfície da bacia hidrográfica, necessária para a produção do volume determinado.
\end{abstract}

Palavras-chave: Plisto-holocénico, Modelo geomorfológico, Modelação geológica 3D, Reconstrução paleoambiental, Sistemas de Informação Geográfica.

Abstract:

Geological modeling of the Late Pleistocene-Holocene sedimentary infill of Pederneira paleolagoon The paleoenvironmental reconstruction of the Portuguese coastal wetlands is a key element for understanding the evolutionary history of the coastal zone in the recent geological past (Quaternary).

Aiming to improve the evolutionary model of Nazaré lowland (Pederneira paleolagoon) during the late Pleistocene and Holocene, a 3D geological modeling approach was used to define the geometry and volume of the main geological units of its sedimentary infill.

With this purpose, three lithostratigraphic units were defined (basal fluvial, marine / lagoon and recent fluvial) and a simple conceptual geomorphological model was developed to overcome the scarcity of geological data and its non-uniform distribution across the study area.

Using spatial interpolation tools in a GIS environment, the paleosurface that accommodate the Pleisto-Holocene sedimentary infill, as well the paleosurfaces that limit their constituent units, were defined. Through 3D geological modeling, it was possible to define the geometry and the total volume of the lithostratigraphic units, and evaluate the reasonableness of the estimated values through the comparison with watershed erosion estimates.

The methodology used in the 3D modeling, produced satisfactory results, providing an effective tool in the problem of paleoenvironmental reconstruction in areas with scarce geologic information.

Keywords: Pleisto-Holocene, Geomorphologic model, 3D Geologic modeling, Paleoenvironmental reconstruction, Geographic Information Systems.

Received: 24 july, 2014; Accepted: 20 November, 2014

\section{INTRODUÇÃ̃O}

As zonas húmidas litorais constituem áreas privilegiadas para o estudo da evolução geomorfológica do litoral e dos fatores que nela intervêm. Ao longo das duas últimas décadas foi desenvolvido, por diversos autores, um modelo conceptual de evolução da zona costeira do território continental português desde o Último Máximo Glaciar, relacionando-o com os respetivos fatores forçadores (globais e regionais), de que se salientam a variação do nível médio do mar, as alterações climáticas e a influência antrópica (e.g., HENRIQUES 1996; FREITAS \& ANDRADE 1997, 1998; BAO et al. 1999; DiAs et al. 2000; CRUCES 2001; CEARRETA et al. 2002; DRAGO et al. 2002; FreitAs et al. 2002; HENRIQUES et al. 2002; CEARRETA et al. 2003; FREITAS et al. 2003a, 2003b; HENRIQUES 2003; SANTOS \& GOÑI 2003; DiNIS \& COSTA 2004; FERREIRA et al. 2004; HENRIQUES \& DINIS 2006; ALDAY et al. 2006; CABRAL et al. 2006; CRUCES et al. 2006; DINIS et al. 2006; DINIS \& TAVARES 2006; DRAGO et al. 2006; FREITAS 2006; CEARRETA et al. 2007; FREITAS et al. 2007; NAUGHTON et al. 2007a,

\footnotetext{
${ }^{(1)}$ Departamento de Geologia, Faculdade de Ciências, Universidade de Lisboa, 1749-016 Lisboa, Portugal. vplopes@fc.ul.pt

${ }^{(2)}$ Centro de Geologia da Universidade de Lisboa.

${ }^{(3)}$ Instituto Dom Luís.
} 
2007b; FERREIRA et al. 2009; LEORRI et al. 2009; FREITAS et al. 2010; HENRIQUES et al. 2010; MOREIRA et al. 2010; CABRAL et al. 2011; HENRIQUES 2013).

A várzea da Nazaré constitui um objeto de estudo de excecional importância, não só pelo facto de ter evoluído a partir de um ambiente lagunar (a antiga laguna da Pederneira), hoje completamente colmatado, como por existir abundante informação histórica e arqueológica sobre a zona, possibilitando assim a eliminação de um hiato de informação significativo sobre a evolução recente dos sistemas sedimentares de transição da região central do litoral ocidental português (FREITAS 2006; FREITAS et al. 2010).

Neste sentido, a várzea da Nazaré foi alvo de estudo num projeto financiado pela Fundação para a Ciência e Tecnologia intitulado "Evolução paleoambiental da planície litoral a sul da Nazaré desde o Tardiglaciar (PaleoNaz)" - PTDC/CTEGEX/65789/2006. No âmbito do referido projeto, foi realizado um estudo exaustivo do ponto de vista sedimentológico, geoquímico e paleontológico de três sondagens mecânicas efetuadas no interior da Várzea da Nazaré e reavaliado um número significativo de relatórios de sondagens anteriormente realizadas no âmbito da construção de estradas e obras de arte que intersectam a referida várzea. Foram assim consideradas três grandes unidades litoestratigráficas que constituem o enchimento sedimentar plistoholocénico, o que permitiu propor uma história evolutiva para esta área e integrá-la no modelo conceptual de evolução da linha de costa ocidental Portuguesa desde o Último Máximo Glaciar, o qual tem vindo a registar sucessivas atualizações, resultado de vários trabalhos de caracterização geomorfológica e sedimentar.

O objectivo deste trabalho é efectuar modelação geológica 3D recorrendo a ferramentas de interpolação espacial no sentido de definir a geometria da paleosuperfície de acomodação do enchimento sedimentar plisto-holocénico e das paleosuperfícies que separam as suas várias unidades litoestratigráficas

\section{ENQUADRAMENTO DA ÁREA DE ESTUDO}

A Várzea da Nazaré localiza-se na costa ocidental Portuguesa, no extremo norte do troço compreendido entre a Nazaré e o Cabo Carvoeiro, o qual representa uma célula costeira de aproximadamente $40 \mathrm{~km}$ de comprimento. A área em estudo está situada entre a Nazaré e a praia do Salgado (Fig. 1).

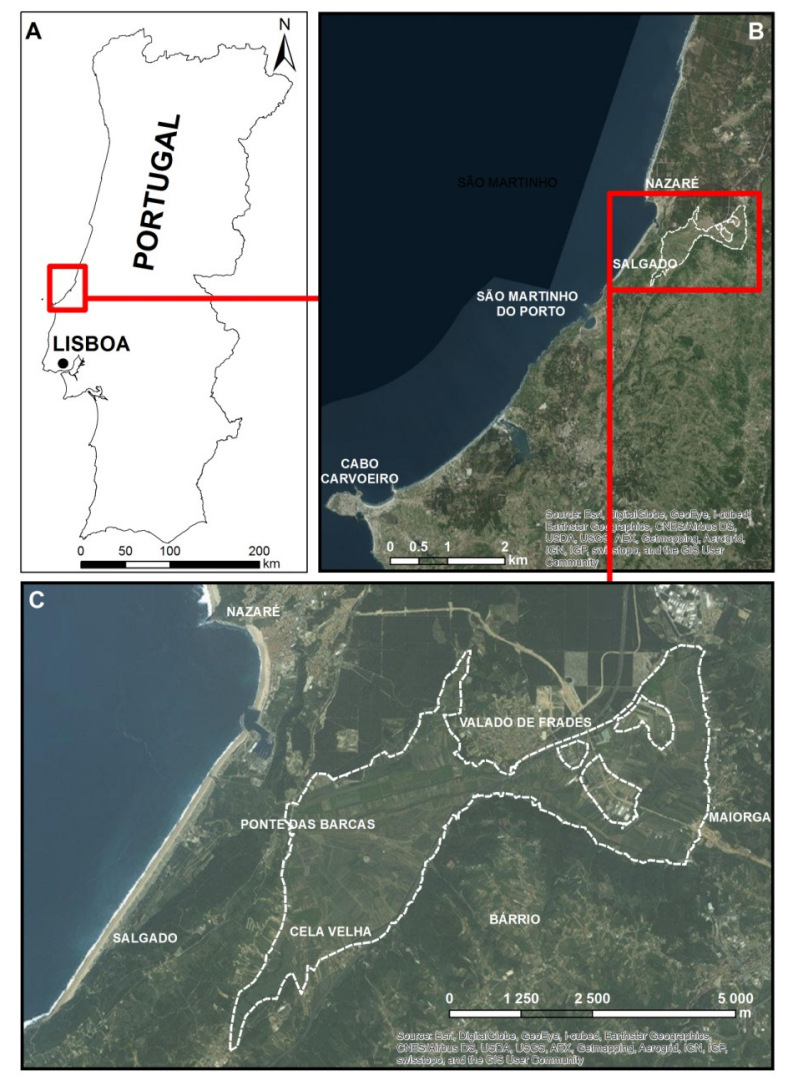

Fig. 1. Localização da paleolaguna da Pederneira. A - no litoral centro de Portugal; B - No troço litoral Nazaré-Cabo Carvoeiro; C - No troço litoral Nazaré-praia do Salgado, a linha tracejada indica o perímetro da paleolaguna da Pederneira.

Fig. 1. Location of the Pederneira paleolagoon. A - In the central coast of Portugal; B - In the coastal section Nazaré - Carvoeiro Cape; C - In the coastal section Nazaré-Salgado beach, the dotted line indicates the Pederneira paleolagoon perimeter. 
Corresponde a $16 \mathrm{~km}^{2}$ de superfície, com cotas decrescentes para poente, geralmente inferiores a $10 \mathrm{~m}$ (NMM - nível médio do mar), podendo chegar a cotas próximas de $23 \mathrm{~m}$ nos bordos; é constituída por sedimentos aluviais detríticos debitados por uma rede de drenagem pertencente a uma bacia hidrográfica com 420 $\mathrm{km}^{2}$ (Fig. 2A) que termina na vertente $\mathrm{W}$ da Serra dos Candeeiros (FREITAS et al. 2010).

Esta superfície representa uma planície aluvial que está relacionada com a secção inferior de vários rios (Fig. 2B): o Rio Alcobaça, resultante da confluência dos Rios Alcôa e Baça; o Rio do Meio, troço jusante do Rio de S. Vicente e Rio da Areia, troço jusante do Rio de Cós (FrEITAS et al. 2010), e ainda pelas ribeiras de Famalicão e de Águas Belas.

A referida superfície apresenta uma forma irregular herdada de constrangimentos tectónicos devido à sua localização no interior da depressão diapírica das Caldas da Rainha e a sua associação à fratura de Fervença (HENRIQUES \& DINIS 2006).
No geral, o bordo sul é bem marcado na morfologia pelo contato entre os calcários mesozóicos dobrados e os sedimentos aluviais horizontais. O bordo norte é em geral menos abrupto, definido pelo contacto entre os materiais detríticos jurássicos ou pliocénicos e as dunas recentes (HENRIQUES 1996). Esta planície aluvial compreende dois setores (Fig. 2B):

- o setor oeste (A) com $10 \mathrm{~km}^{2}$ e um eixo maior de $7.6 \mathrm{~km}$, paralelo à linha de costa. É separado da mesma por um estrangulamento rochoso na Ponte das Barcas (com uma largura aproximada de $218 \mathrm{~m}$ ), e a sua superfície encontra-se a uma cota máxima de $5 \mathrm{~m}(\mathrm{NMM})$;

- o setor leste (B) com $7.7 \mathrm{~km}^{2}$, que comunica com o anterior através de outro estrangulamento rochoso de maiores dimensões no Valado de Frades (largura aproximada de $450 \mathrm{~m}$ ). A sua superfície encontra-se a cotas mais elevadas $(5-10 \mathrm{~m})$ e contém colinas de elevação moderada $(>15 \mathrm{~m})$ constituídas por afloramentos pliocénicos e jurássicos (FREITAS et al. 2010).

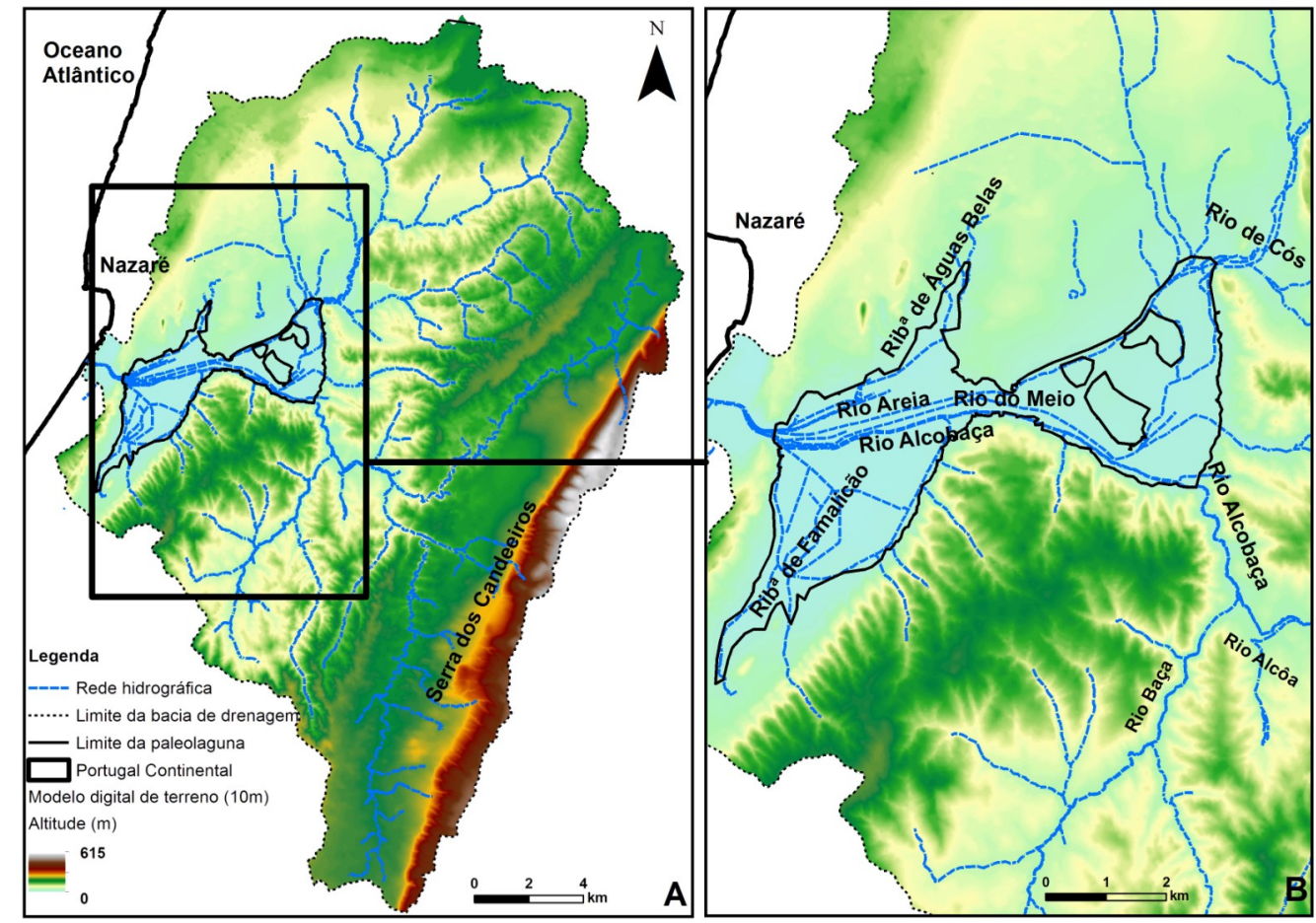

Fig. 2. A - Bacia hidrográfica da várzea da Nazaré; B - Rede hidrográfica da paleolaguna da Pederneira Fig. 2. A - Nazaré plain watershed B - Hydrographic network of Pederneira paleolagoon.

\section{METODOLOGÍA E RESULTADOS}

\subsection{Sedimentologia}

A caraterização do enchimento sedimentar plisto-holocénico foi realizada através do estudo sedimentológico (textura, composição), geoquímico (elementos maiores, menores e traço) e paleoecológico (foraminíferos, ostracodos e nanoplâncton calcário) de 3 sondagens mecânicas com comprimento entre 25 e $30 \mathrm{~m}$ (FREITAS et al. 2010; LOPES 2013), com o objetivo de estabelecer variações de litofácies e definir unidades litoestratigráficas. O enquadramento cronológico foi efetuado com recurso a datações por ${ }^{14} \mathrm{C}$ e OSL. Foram igualmente analisados mais de uma centena de relatórios de sondagens efetuadas para fins geotécnicos (Fig. 3). 


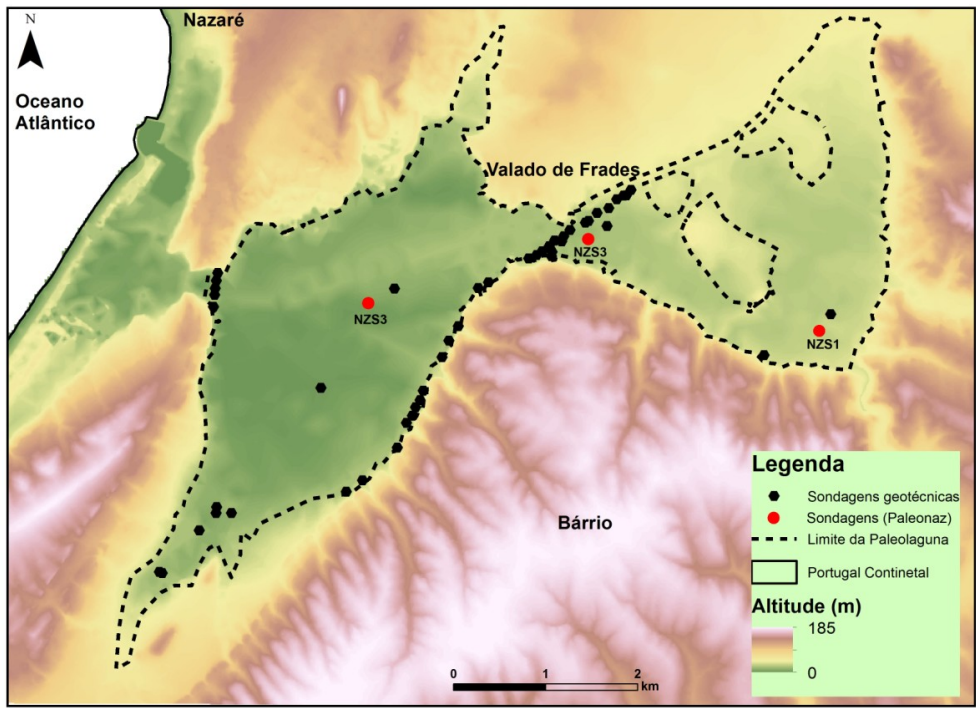

Fig. 3 - Localização das sondagens efetuadas na várzea da Nazaré (marcadores pretos representam as sondagens para fins geotécnicos; marcadores vermelhos representam as sondagens estudadas no âmbito do projeto Paleonaz).

Fig. 3 - Location of cores performed in Nazaré plain (black dots represent the cores performed for geotechnical purposes, red dots represent the cores studied under Paleonaz project).

Através da correlação entre as unidades estabelecidas nas três sondagens e a cronologia associada, foi possível fazer uma reconstrução paleoambiental, tendo sido definidas três grandes unidades litoestratigráficas (Fig. 4).

$\mathrm{Na}$ base está presente a Unidade Fluvial Antiga (Unidade I) caraterizada por areia-areia vasosa, azóica com alguns níveis de cascalho correspondente a uma sedimentação fluvial ocorrida desde o início do Holocénico até cerca de 9700-8500 cal BP. Posteriormente a sedimentação muda, surgindo a Unidade marinho/lagunar (Unidade II), caracterizada por vasa a vasa pouco arenosa orgânica com presença de nanofósseis calcários, fragmentos de concha e foraminíferos/ostracodos. Esta sedimentação indica a presença de um ambiente marinho/estuarino a lagunar que permaneceu até cerca de 5000 cal BP na parte leste e até mais tarde, cerca de 2000 Cal BP, na parte ocidental. Por último, a sedimentação mudou novamente, sendo a unidade de topo (Unidade Fluvial recente - Unidade III) caracterizada por intercalações de areia a areia vasosa com vasa a vasa pouco arenosa, orgânica, azóica. Esta última unidade representa a progradação dos materiais fluviais sobre o antigo espaço lagunar (FREITAS et al. 2010; LOPES 2013).

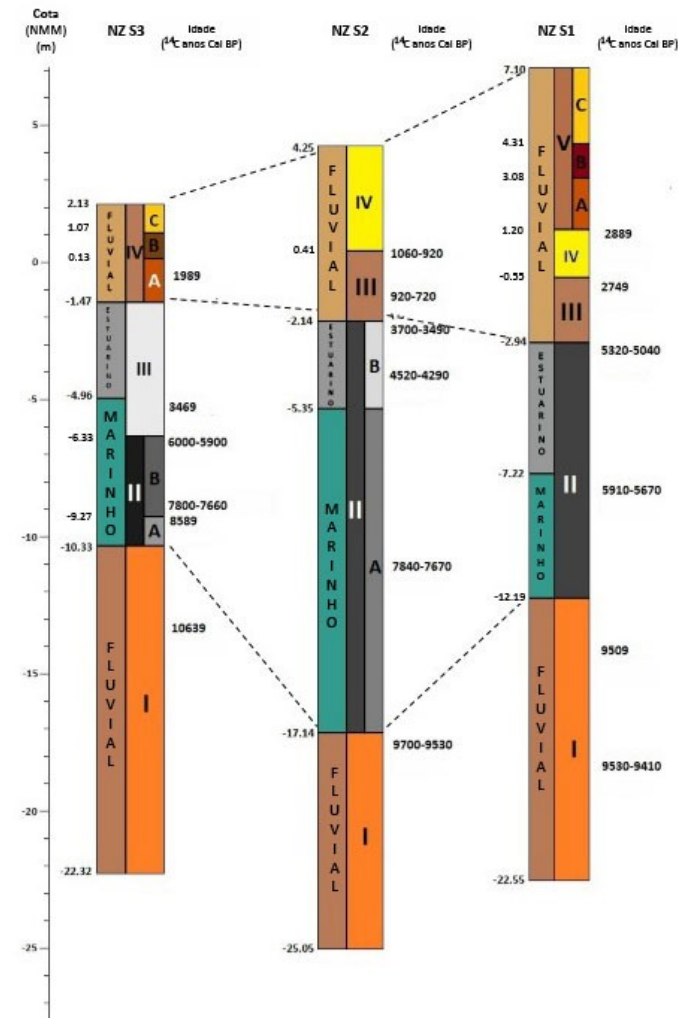

Fig. 4 - Esquema de correlação entre as sondagens NZS1, NZS2 e NZS3 estudadas no âmbito do projeto Paleonaz. Fig. 4 - Correlation scheme between cores NZS1, NZS2 and NZS3 studied within Paleonaz project. 


\subsection{Modelação geológica tridimensional}

Devido à escassez de sondagens e principalmente à sua distribuição espacial não uniforme na área de estudo (Fig. 3), os dados objetivos de localização e profundidade da paleosuperfície de acomodação e configuração da paleorede de drenagem tardiglaciar, mostraram-se inadequados para aplicação direta de procedimentos de interpolação geoestatística. Neste sentido, foi criada informação complementar por forma a constringir a interpolação, através da definição de um modelo geomorfológico concetual, que estabelece o desenho da paleo-rede de drenagem e que assenta em três pressupostos:

1 - O sistema é exorreico e desagua no estrangulamento rochoso mais a oeste;

2 - A incisão de cada talvegue aumenta para jusante;

3 - O sistema estava em equilíbrio com o nível de base.

De acordo com vários autores (HACK 1957; LEOPOLD et al. 1964; SPARKS 1972; CHRISTOFOLETTI 1980; MORISAWA 1985; SCHEIDEGGER 1991, HUGGET 2003), um rio que apresente estas condições desenvolve um perfil longitudinal característico, de forma côncava virada para cima, com declives elevados na cabeceira e que decrescem para jusante até ao nível de base. Para além desta descrição qualitativa, existem diversos estudos que tentaram estabelecer uma descrição matemática para o perfil longitudinal dos rios em equilíbrio, e, no presente estudo, foi adotada a descrição de Green (1934, em CHRISTOFOLETTI 1980):

$$
y=a-k \cdot \log (p-x)
$$

em que $y$ se refere à cota do talvegue relativa ao nível de base num ponto à distância $\mathrm{x}$ da embocadura, $a$ e $k$ são constantes empíricas e $p$ é o comprimento do talvegue.

\subsubsection{Localização do paleotalvegue}

Para definir a planimetria da paleo-rede de drenagem, recorreu-se ao software ArcGIS (v.10). Foi criado um Modelo Digital de Terreno (MDT) da bacia hidrográfica associada à paleolaguna da Pederneira e a rede de drenagem com base em informação cartográfica na escala de 1:25000. Foi registada uma elevada coincidência entre a rede de drenagem criada (em ambiente SIG) e a observada no terreno, ocorrendo resultados menos satisfatórios na planície aluvial devido à intensa intervenção humana aí existente, para fins agrícolas. Foram definidos 4 pontos de referência na interseção entre cada linha de água principal e o limite da paleolaguna. Procurou-se, em seguida, inferir sobre a configuração da rede de drenagem na área colmatada pelos sedimentos plisto-holocénicos. Para esse efeito, foi utilizada uma combinação de dois métodos (Fig. 5):

1 - Realização de vários perfis transversais nos atuais vales baseados no MDT (modelo digital de terreno) produzido, a partir dos quais foram extrapoladas as inclinações das encostas e o seu ponto de interseção em profundidade foi projetado à superfície;

2 - Determinação, da linha que se encontre a meia distância de encostas opostas da paleolaguna, assumindo que o paleotalvegue era simétrico.

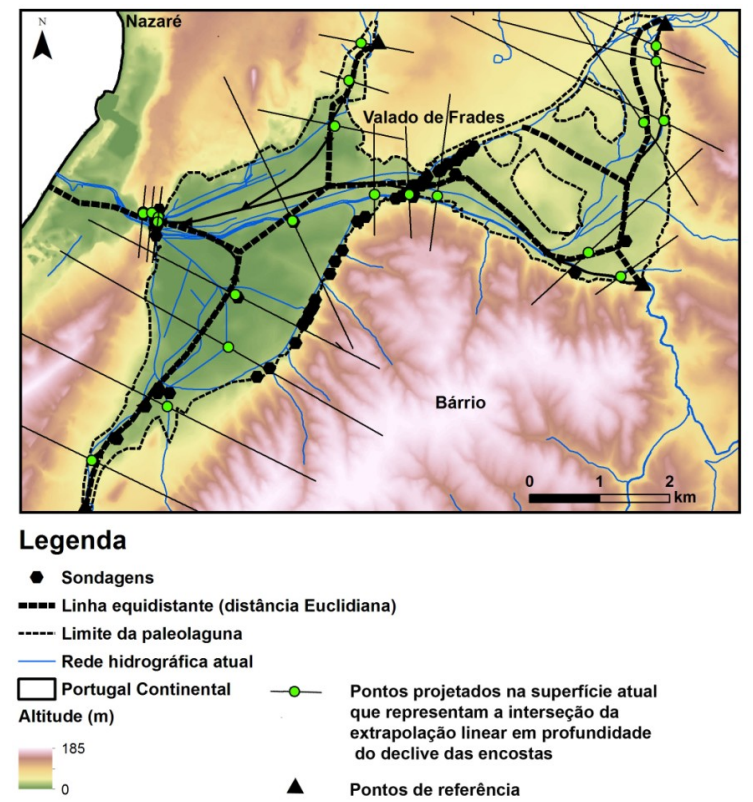

Fig. 5. Figura ilustrativa dos vários métodos aplicados para a determinação da configuração da paleo-rede de drenagem na planície aluvial da Pederneira.

Fig. 5. Illustration of the two methods used to determine the configuration of the Pederneira paleo-drainage. 
Estes resultados foram ajustados sempre que os mesmos se mostraram contraditórios ou que os dados de campo se mostraram insuficientes. $\mathrm{O}$ ajuste foi efetuado com base no contexto geomor- fológico da área de estudo.

A linha de paleotalvegue resultante é composta por um segmento principal que atravessa os dois setores e três tributários menores (Fig. 6).
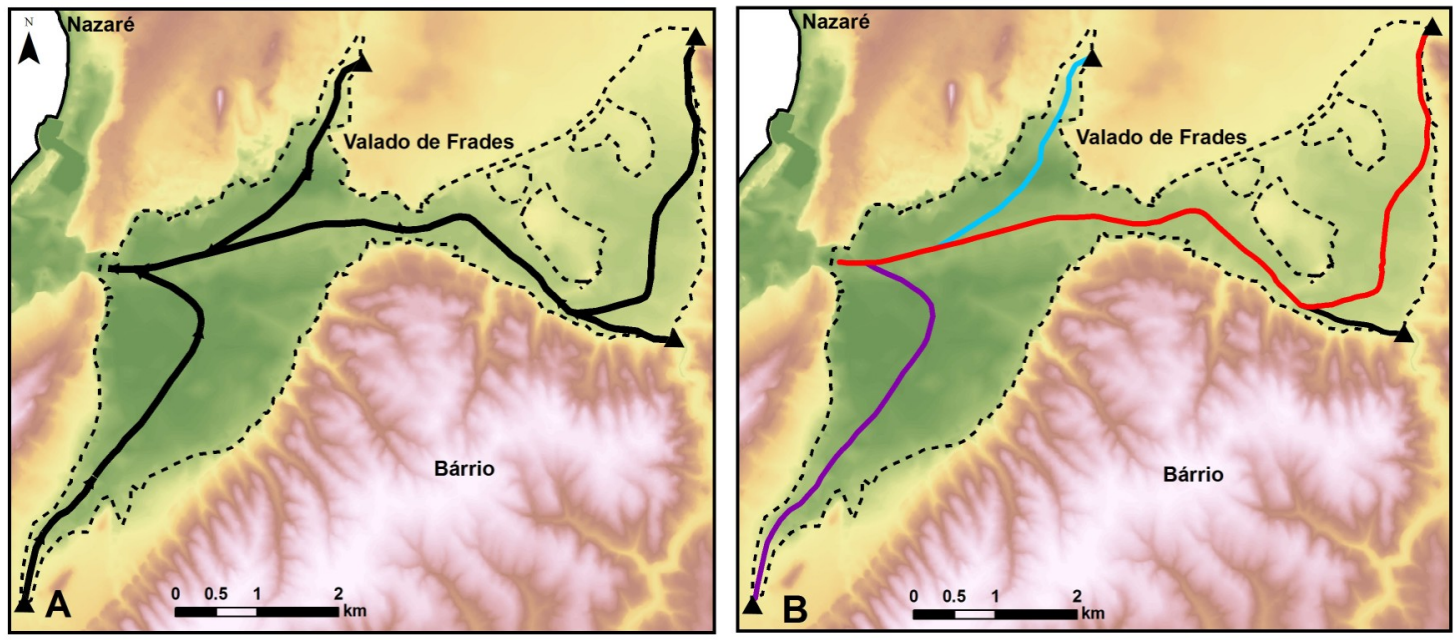

Legenda

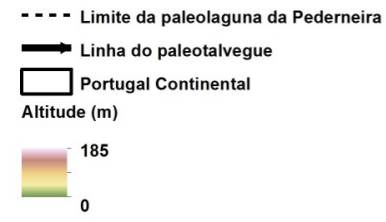

Legenda

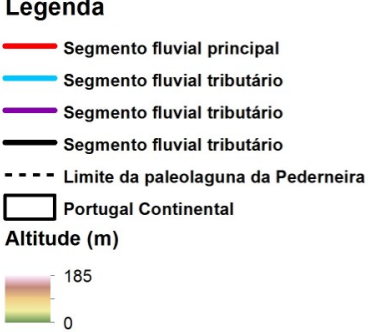

- Ponto de referência

Fig. 6. A - Configuração possível da paleo-rede de drenagem no interior da paleolaguna da Pederneira. B - Composição da paleorede de drenagem no interior da paleolaguna da Pederneira.

Fig. 6. A - Possible configuration of the Pederneira paleo-drainage; B - Different tributaries.

\subsubsection{Atribuição de valores de elevação}

Após a definição planimétrica da linha de paleotalvegue foi determinada a respetiva elevação. Para cada segmento, foram utilizados todos os pontos vizinhos com informação altimétrica conhecida e projetados esses dados num gráfico que relaciona a elevação com a correspondente distância à embocadura. Em todos os casos, o ponto mais alto coincide com os pontos de referência anteriormente referidos e o ponto mais baixo com o estrangulamento rochoso (Ponte das Barcas), com o valor de elevação de $-37 \mathrm{~m}$ (cota deduzida a partir dos dados de sondagens). A cada curva foi aplicada a equação anteriormente referida (que traduz o perfil longitudinal do rio). Dado que entre estes dois pontos fixos é possível fazer passar qualquer curva de função logarítmica, a sua curvatura vai depender da variação do parâmetro $(\mathrm{p}-\mathrm{x})$. A determinação da melhor curva (que representa o perfil longitudinal de cada segmento) foi executada por iterações sucessivas fazendo variar o parâmetro referido de modo a que a equação obtida produzisse valores de elevação para cada segmento o mais próximo dos valores reais conhecidos.

\subsubsection{Reconstrução da paleosuperfície plisto- holocénica}

Na posse da planimetria e altimetria da paleo -rede de drenagem, procedeu-se à interpolação da paleosuperfície de acomodação do enchimento sedimentar plisto-holocénico, usando para isso dados dos relatórios de sondagens, dos pontos ao longo da linha de paleotalvegue e dos pontos que limitam a planície aluvial (extraídos do MDT). Para a interpolação da paleosuperfície foi o usado o método do vizinho natural (ArcGIS v.10).

A superfície produzida apresenta um intervalo de valores de elevação entre cerca de -37 e $23 \mathrm{~m}$ e uma rede de drenagem que reflete os constrangimentos estruturais, favorecendo o desenvolvimento de uma curva larga no setor leste com o talvegue encostado ao bordo sul, apresentando uma seção transversal assimétrica. No setor oeste a incisão do talvegue é muito mais pronunciada, embora produzida por tributários mais pequenos. As seções transversais apresentam-se mais simétricas (Figs. 7 e 8 ). 

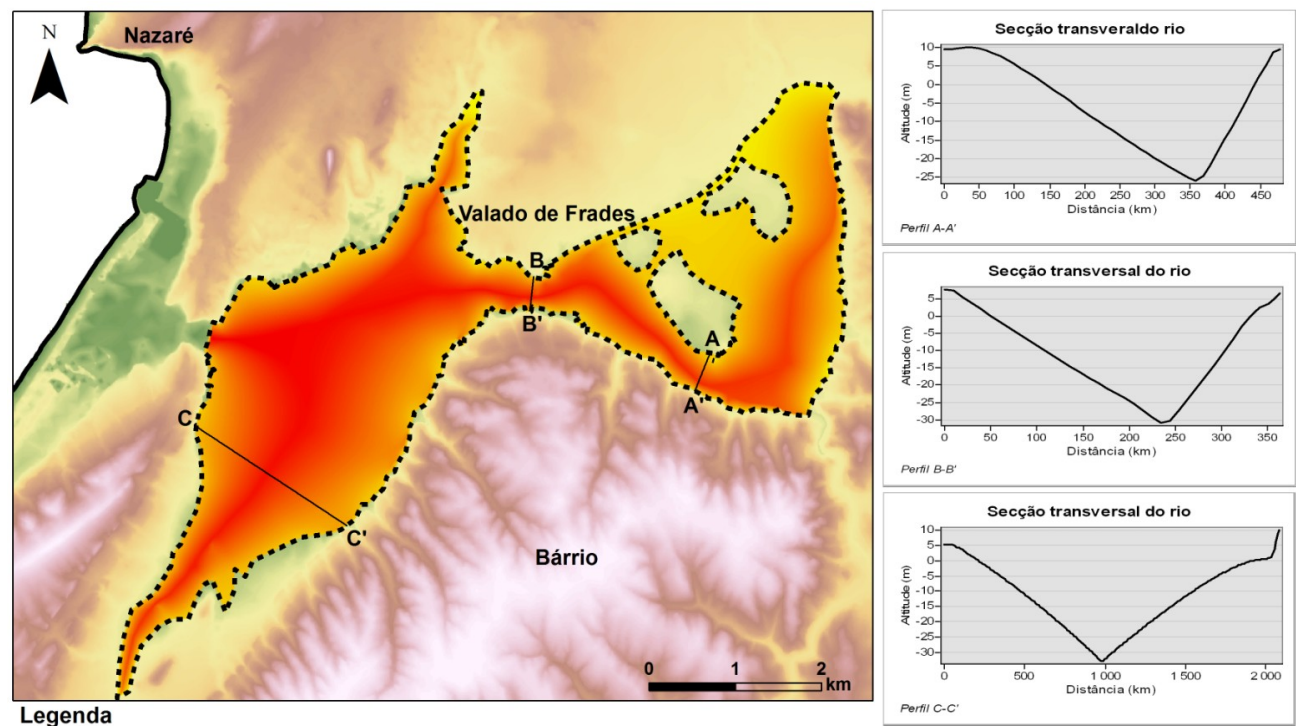

Legenda

...- Limite da paleolaguna da Pederneira Altitude $(m)$

$\square$ Portugal Continental

Paleosuperficie plisto-holocénica

Altitude $(\mathrm{m})$

23

Fig. 7. Superfície A que representa a paleosuperfície de acomodação do enchimento sedimentar plisto-holocénico da paleolaguna da Pederneira e secções transversais às linhas de água .

Fig. 7. Surface A, representing the surface that acomodate the pleisto-holocene infill of Pederneira paleolagoon and crosssections of the valleys.

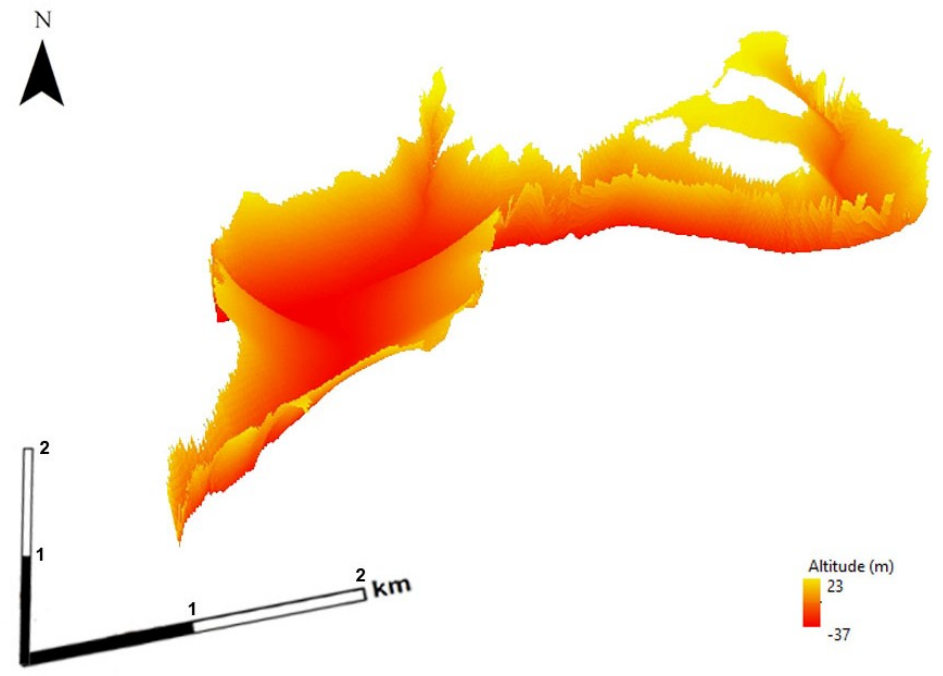

Fig. 8. Paleosuperfície plisto-holocénica visualizada em 3D no programa ArcSene (escala gráfica aproximada).

Fig. 8. 3D view of the pleisto-holocene surface obtained in ArcSene (approximate graphic scale).

\subsubsection{Reconstrução das paleosuperfícies holocénicas}

A reconstrução das superfícies que materializam o limite entre as várias unidades litoestratigráficas do enchimento plisto-holocénico tem, na sua concetualização, um pressuposto diferente do da paleosuperfície plistocénica. Enquanto esta última representa a bacia de acomodação de todo o enchimento sedimentar plisto-holocénico, as primeiras representam os limites que separam as várias unidades litoestratigráficas desse mesmo enchimento sedimentar. A sua reconstrução não integra os mesmos pontos de interpolação nem os mesmos pressupostos referidos no item anterior.

Para a interpolação da paleosuperficie que limita a unidade fluvial antiga da unidade marinha/ lagunar, foram utilizados dados de sondagens. Usou-se Krigagem ordinária (ArcGIS v.10, sem 
efeito de pepita, sem anisotropia e com uma vizinhança padrão, com um máximo de 5 e mínimo de 2 vizinhos).

A superfície resultante apresenta uma forma acanalada, com os valores de elevação entre cerca de -35 e $-4 \mathrm{~m}$, registando-se os valores mais baixos ao longo do traçado do paleotalvegue (Figs. 9 e 10). A unidade Fluvial antiga corresponde a uma sedimentação grosseira alimentada por materiais provenientes da bacia hidrográfica, portanto de origem continental, terrígena. Representa a sedimentação num troço declivoso do rio, que desaguava mais a oeste. Desta forma, devido à sua génese fluvial, faz sentido que esta unidade possa ter configuração de uma camada que reveste o referido paleorelevo, com zonas de sedimentação e outras de erosão resultantes da dinâmica da rede hidrográfica que a gerou. É pois expectável que a superfície que limita superiormente esta unidade tenha uma configuração e geometria herdadas da paleosuperfíce que a antecede e lhe serve de base, que corresponde ao paleorelevo esculpido pela rede de drenagem plistocénica. A superfície obtida apresenta uma configuração bastante plausível e em concordância com o modelo indicado.

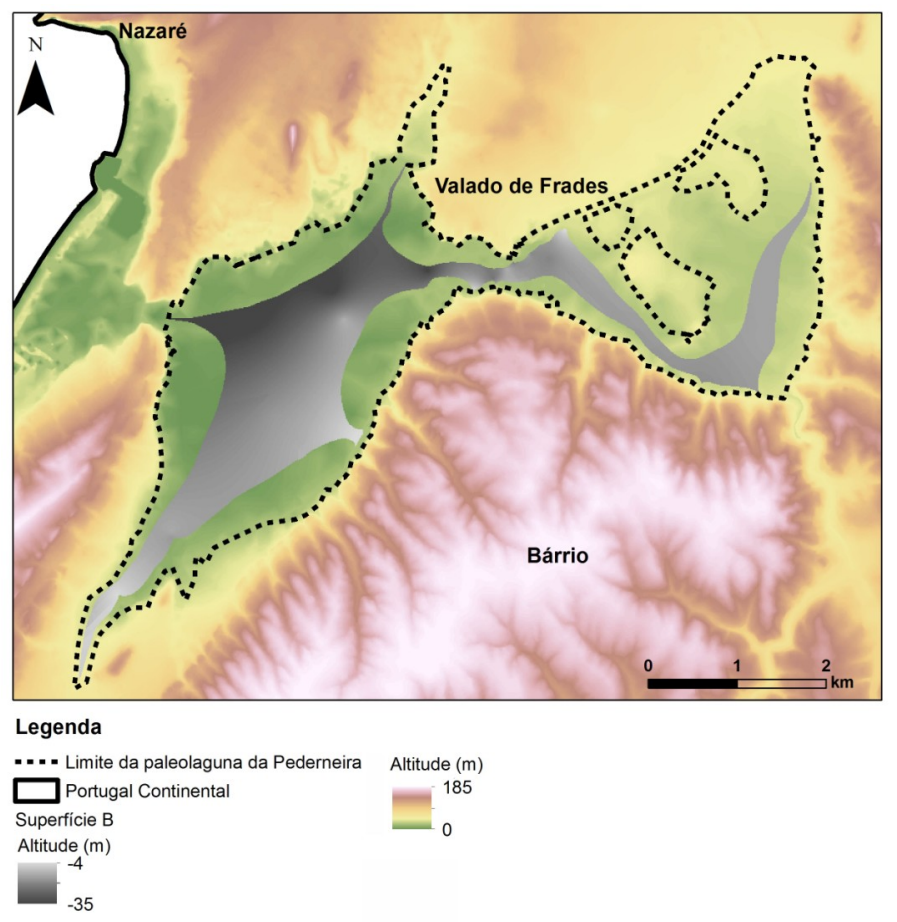

Fig. 9. Superfície B, limite entre a unidade fluvial antiga e a unidade marinho/ lagunar.

Fig 9. Surface B, limit between the basal litostatigraphic unit (fluvial environment) and the intermediate litostatigraphic unit (marine/lagoonal environment).

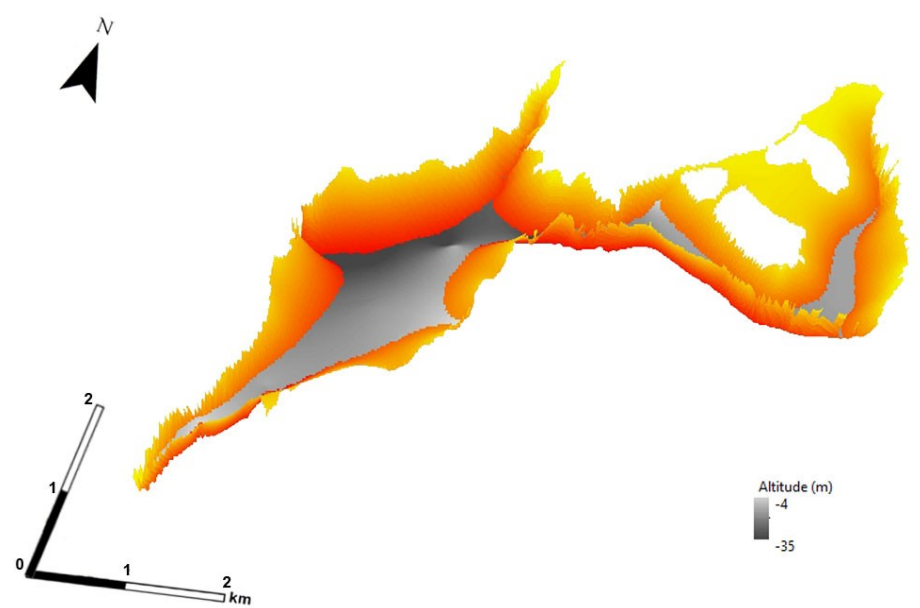

Fig. 10. Superfície B visualizada em 3D no programa ArcSene (escala gráfica aproximada). Fig. 10. 3D view of surface B obtained in ArcSene (approximate graphic scale). 
Para a interpolação da paleosuperficie que limita a unidade marinha/lagunar da unidade fluvial recente foram igualmente utilizados dados de sondagens. Para a interpolação foi utilizada uma superfície de tendência do primeiro grau (ArcGIS v.10).

A superfície produzida apresenta-se subhorizontal, ligeiramente inclinada para leste (em dire- ção a terra), com valores de elevação entre cerca de 4 e $3 \mathrm{~m}$. Esta superfície representa o término da sedimentação marinho/lagunar e o início da sedimentação fluvial recente, sendo plausível uma configuração sub -horizontal (Figs. 11 e 12).

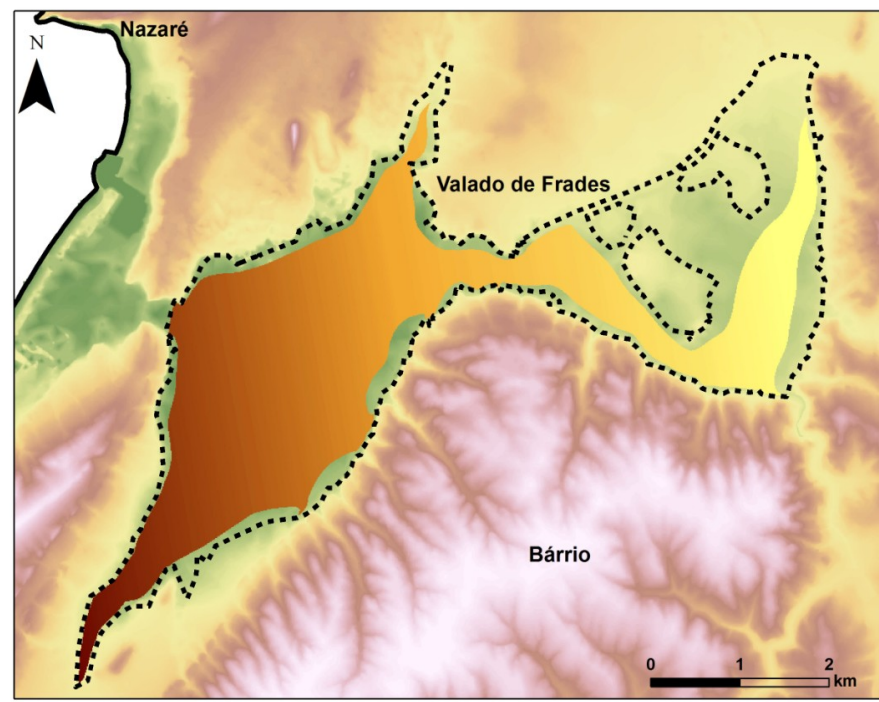

Legenda

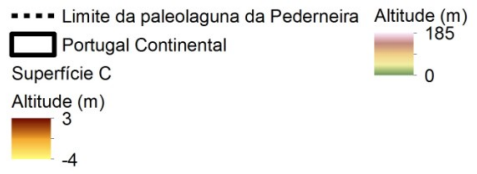

Fig. 11. Superfície C, limite entre a unidade marinho/lagunar e a unidade fluvial recente.

Fig. 11. Surface C, limit between the intermediate unit, marine/lagoonal environment, and the top unit, aluvial environment.

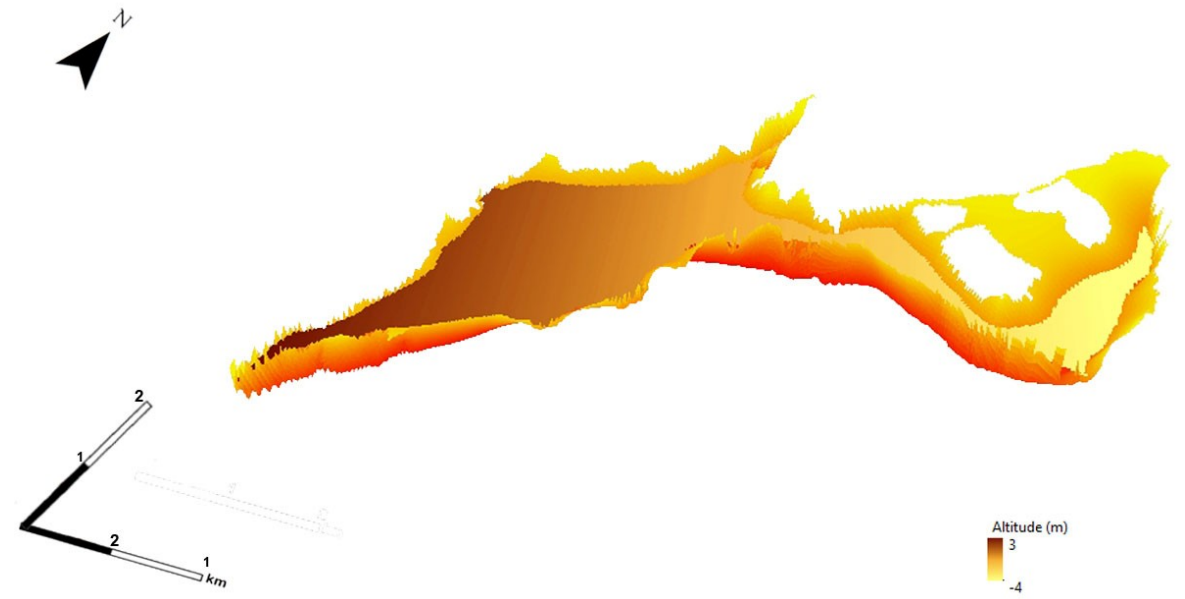

Fig, 12. Superfície C visualizada em 3D no programa ArcSene (escala gráfica aproximada). Fig. 12. 3D view of surface $C$ obtained in ArcSene (approximate graphic scale). 


\subsubsection{Perfis e seções}

Após a definição destas paleosuperficies, foi possível construir perfis e seções que mostram o enchimento sedimentar plisto-holocénico e assim aferir a sua geometria. Da observação destas seções podemos inferir que a unidade mais expressiva é a marinho/lagunar e que as unidades não se distribuem de forma uniforme em toda a área da paleolaguna. Regista-se uma maior expressão da unidade marinho/lagunar no setor oeste, perdendo importância em detrimento da unidade fluvial recente no setor leste. $\mathrm{Na}$ zona do estrangulamento de Valado de Frades nota-se um quase equilíbrio entre estas duas unidades (Fig. 13).

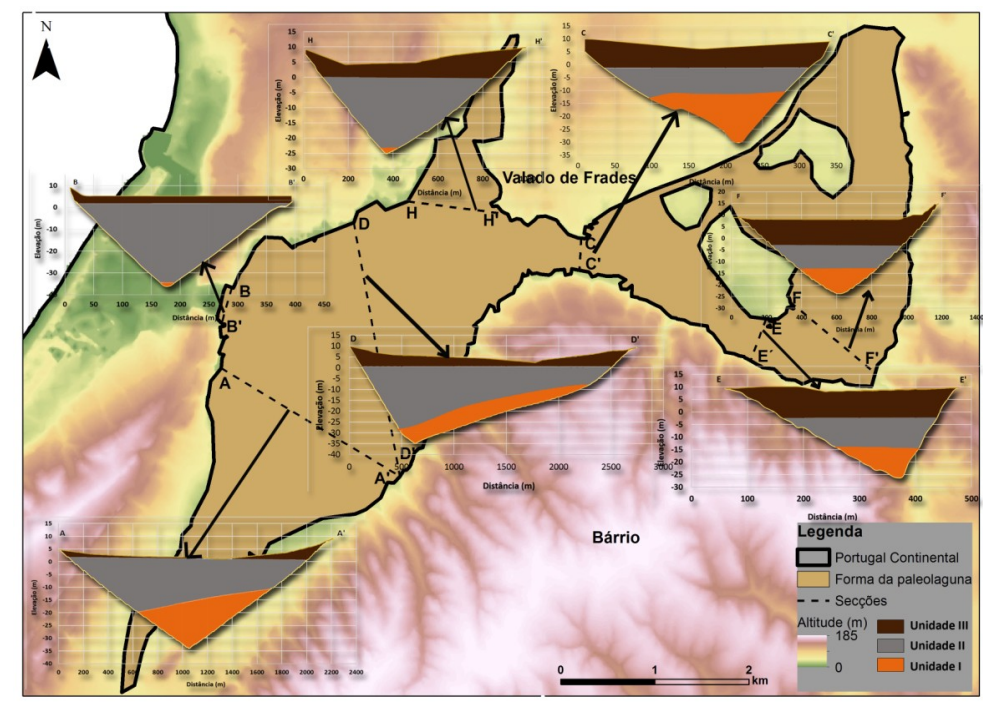

Fig. 13. Representação das várias secções do enchimento sedimentar plisto-holocénico da paleolaguna da Pederneira.

Fig. 13. Sedimentary infill of Pederneira paleolagoon visualized in several cross-sections.

\subsubsection{Volumetria do enchimento sedimentar plisto- holocénico}

Na posse destas paleosuperficies, em ambiente SIG (recorrendo à ferramenta Surface Volume disponível no softawre ArcGIS v.10), foi possível determinar a volumetria associada à totalidade do enchimento sedimentar da paleolaguna da Pederneira bem como das diferentes unidades constituintes (Tabela 1). De acordo com os dados obtidos, o volume total do enchimento sedimentar plistoholocénico da Lagoa da Pederneira corresponde a 265 milhões de $\mathrm{m}^{3}$ de sedimentos. Este volume divide-se pela unidade Fluvial antiga que apresenta uma menor expressão (cerca de 43 milhões de $\mathrm{m}^{3}$ ), seguida pela unidade Fluvial recente com cerca de
78 milhões de $\mathrm{m}^{3}$, sendo a unidade Marinha/ Lagunar a unidade com maior expressão (cerca de 145 milhões de $\mathrm{m}^{3}$ ).

Por forma a aferir a qualidade destes resultados, foi calculado o rebaixamento da superfície da bacia hidrográfica necessário para produzir o volume referido. Os sedimentos constituintes deste depósito sedimentar são oriundos, maioritariamente, da bacia hidrográfica da paleolaguna da Pederneira e uma pequena parte (negligenciável) corresponde a material trazido do oceano durante a transgressão marinha. Neste contexto, determinou-se a taxa de erodibilidade da bacia de drenagem durante o Holocénico. $\mathrm{O}$ volume de sedimentos acumulados $\left(265 \times 10^{6} \mathrm{~m}^{3}\right)$, na paleolaguna da Pederneira,

Tabela 1. Tabela dos volumes das diferentes unidades litoestratigráficas e do volume total da paleolaguna da Pederneira. Table 1. Volume of the different lithostratigrafic units and total sediment volume of Pederneira paleolagoon.

\begin{tabular}{|c|c|c|c|c|}
\hline Unidade litoestratigráfica & $\begin{array}{l}\text { Volume } \\
\left(\mathbf{m}^{3}\right)\end{array}$ & $\begin{array}{c}\text { Volume total }\left(\mathbf{m}^{\mathbf{3}}\right) \\
\text { (obtido da soma dos } \\
\text { volumes das três } \\
\text { unidades) }\end{array}$ & $\begin{array}{c}\text { Volume total }\left(\mathbf{m}^{3}\right) \\
\text { (obtido diretamente } \\
\text { entre a paleosuperfície e } \\
\text { a superfície topográfica } \\
\text { atual) }\end{array}$ & $\begin{array}{c}\text { Diferença } \\
\text { associada à diferença } \\
\text { dos dois volumes totais } \\
(\%)\end{array}$ \\
\hline Unidade III (Fluvial recente) & $7,76 \mathrm{E}+07$ & \multirow{3}{*}{$2,65 \mathrm{E}+08$} & \multirow{3}{*}{$2,66 \mathrm{E}+08$} & \multirow{3}{*}{0,25} \\
\hline $\begin{array}{l}\text { Unidade II (Marinha/ } \\
\text { Lagunar) }\end{array}$ & $1,45 \mathrm{E}+08$ & & & \\
\hline Unidade I (Fluvial antiga) & $4,25 \mathrm{E}+07$ & & & \\
\hline
\end{tabular}


foi dividido pela área da bacia hidrográfica $(420$ $\mathrm{km}^{2}$ ), obtendo-se assim o valor de $0,63 \mathrm{~m}$. Este valor representa o rebaixamento que a superfície da bacia hidrográfica sofreu para produzir o volume de sedimentos do enchimento sedimentar da paleolaguna. Dividindo este rebaixamento pelo tempo que levou a produzi-lo (aproximadamente 10000 anos), obtemos uma taxa de erosão média da bacia hidrográfica de $\sim 0,06 \mathrm{~mm} / \mathrm{ano}$. No entanto, há que ter em conta que nem toda a superfície da bacia hidrográfica contribui de forma igual para o enchimento sedimentar, pois é constituída por formações diferentes, umas mais erodíveis do que outras. Assim, utilizando apenas a área da bacia hidrográfica que corresponde às formações mais erodíveis $\left(275 \mathrm{~km}^{2}\right)$, chegamos ao valor de $\sim 0,1 \mathrm{~mm} /$ ano.

Se compararmos este valor com taxas de erosão determinadas para a mesma tipologia de rochas e de clima na Europa $(0,03 \mathrm{~mm} / \mathrm{ano})$, Ásia $(0,13 \mathrm{~mm} / \mathrm{ano})$ ou Espanha $(0,10-0,30 \mathrm{~mm} / \mathrm{ano})$ (HARRISON 1994; CANTÓN et al. 2001), verificamos que o valor determinado para a área de estudo insere-se na mesma ordem de grandeza dos referidos na bibliografia.

\section{CONCLUSÕES}

A aplicação de técnicas de geoprocessamento, recorrendo a aplicações de Sistemas de Informação Geográfica (ArcGIS v.10,) permitiu, através de interpolação, gerar não só a paleosuperfície de acomodação do enchimento sedimentar plistoholocénico (morfologia e altimetria) da paleolaguna da Pederneira, como também as superfícies que limitam as várias unidades litoestratigráficas consideradas nesse enchimento.

Conclui-se que a unidade basal (unidade Fluvial antiga), que corresponde a um depósito fluvial produzido pela dinâmica da rede de drenagem, é a de menor expressão, com um volume associado de cerca de 43 milhões de $\mathrm{m}^{3}$. A transgressão marinha originou a maior unidade do enchimento sedimentar (unidade Marinha/Lagunar), com cerca de 145 milhões de $\mathrm{m}^{3}$ de sedimentos, formando uma camada sub-horizontal em forma de cunha diminuindo de espessura em direção a terra. Por último, representando uma fácies continental, formou-se a unidade Fluvial recente, também sub-horizontal em forma de cunha diminuindo de espessura em direcção ao mar, apresentando um volume de sedimentos na ordem dos 78 milhões de $\mathrm{m}^{3}$. A volumetria total corresponde a cerca de 265 milhões de $\mathrm{m}^{3}$, resultado de uma taxa média de erosão da bacia hidrográfica de cerca de $0,06 \mathrm{~mm} / \mathrm{ano}$.

A metodologia usada para a modelação tridimensional geológica do enchimento sedimentar da paleolaguna da Pederneira, produziu resultados satisfatórios e mostrou-se uma ferramenta eficaz na problemática da reconstrução paleoambiental em zonas com pouca informação geológica de base.

\section{AGRADECIMENTOS}

Este trabalho é uma contribuição do projecto de I\&D PTDC/CTE-GEX/65789/2006 - Evolução paleoambiental da planície litoral a sul da Nazaré desde o Tardiglaciar (PaleoNaz) financiado pela FCT.

\section{BIBLIOGRAFIA}

Alday, M.; Cearreta, A.; Cachão, M.; Freitas, M.C.; ANDRADE, C. \& GAMA, C. 2006. Micropaleontological record of Holocene estuarine and marine stages in the Corgo do Porto rivulet (Mira River, SW Portugal). Estuarine, Coastal and Shelf Science, 66: 532-543.

BaO, R.; Freitas, M.C. \& ANDRAdE, C. 1999. Separating eustatic from local environmental effects a late holocene record of coastal change in Albufeira lagoon, Portugal. The Holocene, 9 (3): 341-352.

Cabral, M.C.; Freitas, M.C.; Andrade, C.; Moreira, S. \& CRUCES, A. 2011. Holocene ostracods of Pederneira (Nazaré, Portugal), a structurally-segmented infilled lagoon. JOANNEA GEOLOGIE UND PALÄONTOLOGIE, 11: 36-38.

Cabral, M.C.; Freitas, M.C.; Andrade, C. \& Cruces, A. 2006. Coastal evolution and Holocene Ostracods in Melides lagoon (SW Portugal). Marine Micropaleontology, 60: 181-204.

CAntón, Y.; Domingo, F.; Solé-Benet, A. \& Puig DE FÁBREGAS, J. 2001. Hydrological and erosion response of a badlands system in semiarid SE Spain. Journal of Hydrology, 252: 65-84.

Cearreta, A.; Alday, M.; Freitas, M.C. \& Andrade, C. 2007. Postglacial foraminifera and paleoenvironments of the Melides lagoon (SW Portugal): towards a regional model of coastal evolution. Journal of Foraminiferal Research, 37, 2: 125-135.

Cearreta, A.; Alday, M.; Freitas, M.C.; Andrade, C. \& CRUCES, A. 2002. Modern and Holocene foraminiferal record of alternating open and restricted environmental conditions in the Santo André lagoon, SW Portugal. Hydrobiologia, 475/476: 21-27.

Cearreta, A.; Cachão, M.; CABral, M.C.; BaO, R. \& RamaLHO, M.J. 2003. Lateglacial and Holocene Environmental Changes in Portuguese coastal lagoons: 2 Microfossil multiproxy reconstruction of the Santo André coastal area. The Holocene, 13, 3: 447-458.

Christofoletti, A. 1980. Geomorfologia. 2a. Ed. São Paulo. Edgard Blucher Lda.

CRUCES, A. 2001. Estudo a micro e meso-escala temporal de sistemas lagunares do SW alentejano (Portugal): as lagunas de Melides e Santo André. Tese de mestrado. Universidade de Lisboa.

Cruces, A.; Freitas, M.C.; Andrade, C., Munhá, J.; Tassinari, C.; Vale, C. \& Jounanneau, J.-M 2006. The importance of geochemistry in multidisciplinary studies of lagoonal environments at different time scales: the case of Santo André lagoon (SW Portugal). Journal of Coastal Research, SI 39: 1716-1722.

Dias, J. M. A.; Boski, T.; Rodrigues, A. \& Magalhães, F. 2000. Coastline evolution in Portugal since the Last Glacial Maximum until present - a synthesis. Marine Geology, 170: 177-186. 
Dinis, J. \& CostA, P. J. M. 2004. Holocene infill of the Caldas da Rainha Valley. Anthropological and natural impacts in the fast evolution of three coastal lagoons. In Leroy S. and Costa P. (eds.), ICSU Dark Nature - IGCP 490, Mauritania, 1st joint meeting Mauritania: the desert and the coast. MauritaniaVolume of abstracts and field guide: 63-66.

Dinis, J. \& TAVARES, A. 2006. Condicionantes naturais e antrópicas na evolução holocénica de 3 lagunas do centro de Portugal (Óbidos, Alfeizerão e Pederneira). Actas do $2^{\circ}$ Seminário sobre Sistemas Lagunares Costeiros. Vila Nova de Santo André, Biblioteca Municipal, 2006. Escola Superior de Educação João de Deus: $25-41$.

Dinis, J. L.; Henriques, V.; Freitas, M.C.; Andrade, C. \& CosTA, P. 2006. Natural to anthropogenic forcing in the Holocene evolution of three coastal lagoons (Caldas da Rainha valley, western Portugal). Quaternary International, 150: 41-51.

Drago, T.; Freitas, C.; Rocha, F.; Cachão, M.; Moreno, J.; Naughton, F.; FradiQue, C.; AraúJo, F.; Silveira, T.; Oliveira, A.; Cascalho, J. \& FAteLA, F. 2006. Paleoenvironmental evolution of estuarine systems during the last 14000 years - the case of Douro estuary (NW Portugal). Journal of Coastal Research, SI 39: 186-192.

Drago, T.; Naughton, F.; Moreno, J.; Rocha, F.; CACHÃO, M.; SANCHEZ GoÑI, M.F.; OliveIRA, A.; Cascalho, J.; Fatela, F.; Freitas, C. \& AndRADE, C. 2002. Geological record of environmental changes in the Douro estuary (NW Portugal), since the Late Glacial. Proceedings, LITTORAL 2002, Eurocoast, Vol. III: 341-346.

Ferreira, T.; Freitas, M.C.; Cruces, A. \& Andrade, C. 2004. Lagoas Interdunares - exemplos do Litoral SW Alentejano. Livro de resumos, $1^{\circ}$ Seminário de Sistemas Lagunares Costeiros, Santo André: p. 15.

Ferreira, T.; RAmos, R.; Freitas, M.C. \& ANDRADE, C. 2009. Morphological evolution of the Óbidos lagoon (western coast of Portugal) since the Holocene transgressive maximum. Journal of Coastal Research, SI 56, V. I: 612-616.

FREITAS, C. \& ANDRADE, C. 1998. Evolução do litoral português nos últimos 5000 anos. Alguns exemplos. Almadan, Centro de Arqueologia de Almada: 6470.

Freitas, M. C. 2006. A Lagoa da Pederneira no contexto da evolução Holocénica do Litoral Centro. Colóquio "Lagoa da Pederneira: Geologia e História", Câmara Municipal da Nazaré: 2 p.

Freitas, M.C.; Andrade, C.; Ferreira, T.; Cruces, A. \& ARAÚJO, M.F. 2007. Wet dune slacks, sea-level and coastal evolution in the southwestern Portuguese façade. Journal of Coastal Research, SI 50: 231236.

Freitas, M.C. \& ANDRADE, C. 1997. Sistemas fluviolagunares a Sul do Tejo: evolução a escalas temporais diferentes. Colectânea de Ideias sobre a Zona Costeira de Portugal. Associação EurocoastPortugal: 525-545.

Freitas, M.C.; Andrade, C. \& Cruces, A. 2002. The geological record of environmental changes in southwestern Portuguese coastal lagoons since the Lateglacial. Quaternary International, vol. 93-94 (C): 161-170.
Freitas, M.C.; Andrade, C.; Ramos, R.; Cruces, A. \& HENRIQUES, V. 2010. Evolução paleoambiental da planície litoral a sul da Nazaré desde o Tardiglaciar. Integração no modelo de evolução do litoral ocidental Português. Proceedings, Iberian Coastal Holocene Paleoenvironmental Evolution, Coastal Hope 2010: 48-58.

Freitas, M.C.; Andrade, C.; Rocha, F.; TAssinari, C.; MunhÁ, J.M.; CRuces, A.; Vidinha, J. \& Silva, C. M. 2003a. Lateglacial and Holocene environmental changes in Portuguese coastal lagoons: 1. The sedimentological and geochemical records of the Santo André coastal area (SW Portugal). The Holocene, 13, 3: 433-446.

Freitas, M.C.; Cachão, M.; Andrade, C. \& Cruces, A 2003b. O ciclo sedimentar do Tardiglaciar e Holocénico. Exemplos do litoral SW português. VI Congresso Nacional de Geologia, Ciências da Terra (UNL), Lisboa, $\mathrm{n}^{\mathrm{o}}$ esp. V, CD-ROM: H61-H64.

HACK, J. T. (1957) - Studies of longitudinal stream profiles in Virginia and Maryland. U. S. Geol. Sur. Prof paper. Washington, EUA. (294-B): 45-97.

HARrison, C. G. A. (1994) - Rates of continental erosion and mountain building. Geol Rundsch, 83: 431-447.

Henriques, M.V. 2013. O litoral dos coutos de Alcobaça. Evolução sedimentar e histórica da Lagoa da Pederneira. In J.A. Carreiras (dir.) Atas de Congresso Mosteiros Cistercienses, História, Arte, Espiritualidade e Património, Alcobaça, Tomo III: 423-442.

HENRIQUES, M.V. \& DINIS, J. 2006. Avaliação do enchimento sedimentar holocénico na planície aluvial da Nazaré (Estremadura Portuguesa). Proceedings X Colóquio Ibérico de Geologia. A geografia Ibérica no contexto Europeu. Évora. PDF079: 16 p.

HENRIQUES, M.V. 2003. Análise morfológica e evolução sedimentar da planície aluvial da Nazaré (Estremadura Portuguesa). Thalassas, 19 (2a): 54 56.

Henriques, M.V. 1996. A Faixa litoral entre a Nazaré Peniche. Unidades geomorfológicas e dinâmica atual dos sistemas litorais. Tese de doutoramento, Universidade de Évora.

Henriques, M.V.; Freitas, M.C.; Ramos, R. \& Andrade, C. 2010. A Lagoa da Pederneira - Evolução geomorfológica e contexto socioeconómico regional. Proceedings, Iberian Coastal Holocene Paleoenvironmental Evolution, Coastal Hope 2010: 67-69.

Henriques, M.V.; Freitas, M.C.; Andrade, C. \& Cruces, A. 2002. Alterações morfológicas em ambientes estuarinos desde o último máximo transgressivo exemplos da Estremadura e Alentejo. Publicações da Associação Portuguesa de Geomorfólogos, vol. I, APGeom, Lisboa: 99-109.

HugGett, R. J. 2003. Fundamentals of Geomorphology. London. Routledge Fundamentals of Physical Geography.

Leopold, B.L.; Wolman, G.M. \& Miller, P.J. 1964. Fluvial processes in geomorphology. San Francisco. W. H. Freeman and Company.

Leorri, E.; Fatela, F.; Moreno, J.; Antunes, C.; CearRETA, A.; Freitas, M.C. \& ANDrade, C. 2009 Intertidal foraminifera in the Mira estuary, SW Portugal, and their use as sea-level proxies. Geogaceta, 46: 71-74. 
LOPES, V.P. 2013. Modelação geológica tridimensional: aplicação à evolução da várzea da Pederneira (Nazaré). Tese de Mestrado, Universidade de Lisboa.

Moreira, S.; Freitas, M.C.; Araúuo, M.F.; Cruces, A.; Andrade, C.; Regala, R. \& Lopes, V. 2010. Paleoenvironmental evolution of Pederneira lagoon (Nazaré, Portugal) using sedimentological and geochemical proxies. Proceedings, Iberian Coastal Holocene Paleoenvironmental Evolution, Coastal Hope 2010: 76-77.

MorisawA, M. (1985) - Geomorphology texts 7, Rivers. New York. Longman Group Ltd.

Naughton, F.; SÁnchez GoÑI, M. F.; Drago, T.; Freitas, M.C. \& OliveirA, A. 2007a. Holocene changes in the Douro estuary (northwestern Iberia). Journal of Coastal Research, 23: 711-720.

NaUghton, F.; SANChez GoÑI, M.F.; Desprat, S.; TURON, J.-L.; Duprat, J.; Malaizé, B.; Joli, C.; Cortijo, E.; Drago, T. \& Freitas, M.C. 2007b. Present-day and past (last 25000 years) marine pollen signal off western Iberia. Marine Micropaleontology, 62: 91114.

SANTOS, L., \& SANCHEZ GoÑI, M.F. 2003. Lateglacial and Holocene environmental changes in Portuguese coastal lagoons 3: vegetation history of the Santo André coastal area. The Holocene, 13, 3: 459-464.

SCHEIDEGGER, E.A. 1991. Theoretical Geomorphology. Third edition. Berlin. Springer-Verlag.

SpARKS, B. W. 1972. Geomorphology. Second edition. Great Britain. Longman Group Ltd. 